\title{
QUALIDADE DE VIDA RELACIONADA À SAÚDE: ANÁLISE DIMENSIONAL DO CONCEITO
}

\author{
Lara Borges de Vasconcelos', Míria Conceição Lavinas Santos1, Raimunda Magalhães da \\ Silva1, Carlos Garcia Filho1, Victor Lavinas Santos1 e Débora Rodrigues Guerra Probo1
}

1Universidade de Fortaleza (Unifor), Fortaleza, Brasil. laraborgesvasconcelos@hotmail.com; miriaclavinas@gmail.com; msilva@unifor.br; victorls380@gmail.com; deboraa@unifor.br

\begin{abstract}
Resumo. Introdução: A qualidade de vida relacionada à saúde foi escolhida para compor o foco deste estudo porque implica a importância do aprofundamento de significado dos conceitos envolvidos e enfatiza a oportunidade de estudá-los como temas socialmente construídos. Objetivo: Analisar o conceito de qualidade de vida relacionado à saúde através do Modelo de Análise de Conceito Dimensional. Método: De viés analítico, este estudo descreve a construção social e lógica do referido conceito, diferencia as duas perspectivas e identifica e examina as proposições. Resultado: Na atualidade, o termo "qualidade de vida" ganha maior fundamentação teórica e metodológica. As suas definições são classificadas como Global Tipo I, II e III. A função do instrumento utilizado é discriminar, descrever e predizer o nível de qualidade de vida das pessoas, baseando-se nos diversos aspectos da qualidade de vida, de acorco com o contexto. Conclusão: A medida da qualidade de vida depende do consenso entre os especialistas, sendo necessário o debate contínuo sobre o constructo deste conceito.
\end{abstract}

Palavras-chave: Qualidade de Vida; Formação de Conceito; Classificação; Saúde; Modelos Teóricos.

\section{QUALITY OF LIFE RELATED TO HEALTH: DIMENSIONAL ANALYSIS OF THE CONCEPT}

Abstract. Introduction: Health-related quality of life was chosen to form the focus of this study because it implies the importance of deepening the meaning of the concepts involved and emphasizes the opportunity to study them as socially constructed themes. Objective: To analyse the concept of health-related quality of life through the Dimensional Concept Analysis Model. Method: Analytically, this study describes the social and logical construction of that concept, differentiates the two perspectives and identifies and examines the propositions (hypotheses). Result: Currently, the term "quality of life" gains greater theoretical and methodological foundation. Its definitions are classified as Global Type I, II and III. The function of the instrument used is to discriminate, describe and predict people's level of quality of life, based on the various aspects of quality of life, according to the context. Conclusion: The measure of quality of life depends on consensus among specialists, requiring a continuous debate on the construct of this concept.

Keywords: Quality of Life; Concept Formation; Classification; Health; Theoretical Models.

\section{INTRODUÇÃO}

No século XXI, a base conceitual da qualidade de vida relacionada à saúde tem ganhado maior fundamentação teórica e metodológica, a partir do aprimoramento de pesquisas realizadas nas últimas décadas. A maioria delas voltada para a produção de conhecimento da área da saúde, das questões sociais, individuais e ambientais como resultado das políticas públicas em relação às práticas promotoras da saúde e do bem-estar da vida 
individual e coletiva (Minayo, Hartz, \& Buss, 2000; Viana \& Sampaio, 2019; Vilarta, Gutierrez, \& Monteiro, 2010).

Um importante aspecto a ser considerado é o termo "qualidade de vida relacionada à saúde". Ele traz a intersecção entre dois conceitos - qualidade de vida e saúde - que se desvelam e relevam não apenas através do processo saúde-doença, mas também, e sobretudo, como um processo de viver essencialmente humano. Essa intersecção promove abordagens integradoras e interdisciplinares, compreendidas por Garratt, Schmidt, Mackintosh e Fitzpatrick (2002) como frutos de uma construção subjetiva e multidimensional ao mesmo tempo.

Portanto, a análise da qualidade de vida relacionada à saúde foi escolhida para compor este estudo porque compreende a importância do aprofundamento de significado dos conceitos e possibilita pensá-los como temas socialmente construídos - ou seja, como temas que variam a depender das perspectivas e dos contextos. Não obstante sua plasticidade, tratase de um conceito que necessita ser devidamente descrito e compreendido, de forma clara e explícita, possibilitando a sua operacionalização em análises científicas.

Nesse sentido, o objetivo deste artigo é analisar o conceito de qualidade de vida relacionada à saúde através do Modelo de Análise de Conceito Dimensional (Caron \& Bowers, 2000).

\section{METODOLOGIA}

Trata-se de um estudo analítico do conceito "qualidade de vida relacionada à saúde", tendo como base teórica o Modelo de Análise de Conceito Dimensional (Caron \& Bowers, 2000). Este estudo foi realizado no período de janeiro de 2018 a janeiro de 2020.

Para o embasamento teórico, foi realizada uma ampla revisão da literatura em artigos nas áreas de enfermagem, medicina e psicologia, publicados nos últimos 20 anos, a fim de identificar os estudos que abordam o termo "qualidade de vida e saúde". Para tanto, foram feitas buscas nas bases de dados virtuais LiLacs, Psycinfo, MedLine, Pubmed, Scielo e Cochrane Library; foram usados os termos "qualidade de vida", "qualidade de vida relacionada a saúde" e "saúde", nos idiomas português, inglês e espanhol. Participaram do levantamento dos estudos dois alunos de graduação e um pós-doutorando do Programa de Saúde Coletiva da Universidade de Fortaleza (Unifor). Foram encontrados 3.000 estudos, 
dos quais selecionou-se 40, por terem correlação direta com o tema e a análise proposta, dois quais foram analisados pelos três professores do referido programa.

Posteriormente, foi feita uma leitura compreensiva dos artigos, e buscou-se os elementos atribuídos aos termos referidos. No processo de análise, considerou-se as abordagens e a evolução histórica dos conceitos de qualidade de vida e saúde propostos na literatura.

A princípio o modelo foi desenvolvido como uma abordagem metodológica para a base da teoria em pesquisa qualitativa, posteriormente estendida para uma teoria mais geral da análise. Nele estão incorporadas as seguintes suposições: a realidade é (1) socialmente construída, (2) geralmente definida a partir de uma perspectiva particular e (3) situada contextualmente (Caron \& Bowers, 2000).

A Análise Dimensional não é um processo linear. Nela, os procedimentos sugeridos ocorrem simultaneamente, compreendendo duas fases. Na primeira, o pesquisador identifica dimensões envolvidas no conceito e o significado atribuído a essas dimensões. Na segunda, o pesquisador integra as dimensões e analisa as relações entre elas; ou seja, analisa como as relações se dão ao longo de perspectivas e contextos, e quais as consequências do uso do conceito.

Quatro passos são essenciais para a construção social de um conceito, segundo Caron e Bowers (2000):

1) Descrever a construção social do conceito em si: requer identificar as dimensões usadas na construção do conceito e descrever as relações entre as diversas dimensões. Perguntar, explorar as maneiras pelas quais o conceito é usado: onde, quando, como, por quem, e as consequências do seu uso.

2) Descrever a lógica do conceito: exige abordar múltiplas perspectivas dentro de múltiplos contextos, com a finalidade de mostrar a situação ou a natureza da situação na qual o conceito foi criado. Para isso, é preciso examinar as suas proposições - isto é, a natureza do conceito -, que podem ser implícitas ou explícitas, consistentes ou coerentes.

3) Diferenciar as relações entre as perspectivas: requer identificar como as diferentes dimensões são integradas no conceito e se há divergência nos objetivos das partes, pois isto pode causar confusão, frustração e dificuldade para se atingir os objetivos. Portanto, deve-se dispensar particular atenção na análise sobre "quem" definiu "o quê" e "como".

4) Identificar e examinar as proposições (hipóteses): exige observar a qual perspectiva as hipóteses estão associadas. Em outras palavras, o pesquisador estabelece quais são as proposições sobrepostas às várias definições dadas de diversas perspectivas. 


\section{RESULTADOS E DISCUSSÃO}

\subsection{Descrever a construção social do conceito}

A definição do termo "qualidade de vida relacionada à saúde" traz dois conceitos, com definições em plena mutação: qualidade de vida e saúde. Apesar da dificuldade enunciada, atualmente há uso generalizado. Ele é utilizado em duas vertentes: na linguagem cotidiana (população em geral, jornalistas, políticos, área de comunicação) e no contexto da pesquisa científica. Neste último, o conceito começou a ser utilizado na década de 1960 (Gaiva, 1998). Os campos do saber que mais o utilizam são a economia, a sociologia, a educação, a medicina, a enfermagem e a psicologia (Seidl \& Zannon, 2004).

$\mathrm{Na}$ área da saúde, o panorama que permitiu o incremento do conceito foi o desenvolvimento de terapias para doenças crônicas, levando, assim, ao aumento da sobrevida para doenças que eram letais no passado - o que resultou na necessidade de conhecer a experiência vivida, em particular as limitações, destes doentes crônicos. Entretanto, apesar de sua difusão mais recente, seu uso já era registrado na literatura médica da década de 1930 (Costa \& Araújo, 2001).

Porém, foi a partir dos anos 1980 que as pesquisas sobre qualidade de vida se tornaram imprescindíveis à área da saúde, visto que seus resultados contribuem para aprovar e definir tratamentos e avaliar custos e benefícios no cuidado prestado (Fayers, 2000; Minayo et al., 2000). Observou-se também, nessa época, o aumento acentuado de instrumentos para a avaliação da qualidade de vida (Costa \& Araújo, 2001).

Segundo Brasel (2007), nas primeiras pesquisas focadas em qualidade de vida, a avaliação era feita por um observador com perguntas objetivas, geralmente um profissional de saúde como no caso do índice Karnofsky, um dos primeiros instrumentos da área médica, criado em 1984, sobre qualidade de vida em pacientes oncológicos. Ainda que de aspecto objetivo, a maioria dos questionários de avaliação da qualidade de vida incorporavam perguntas subjetivas, centradas na percepção pessoal.

A partir da década de 1990, duas dimensões foram destacadas pelos pesquisadores da área: a subjetividade e a multidimensionalidade da qualidade de vida.

A subjetividade trata de considerar a percepção da pessoa sobre o seu estado de saúde e sobre os aspectos não-médicos do seu contexto de vida. É identificada por meio de uma 
avaliação centrada na percepção pessoal da pessoa pesquisada. Os autores abordam a qualidade de vida como uma representação social criada a partir de parâmetros subjetivos (bem-estar, amor, felicidade, prazer e realização pessoal) e objetivos referentes à satisfação das necessidades básicas criadas pelo grau de desenvolvimento econômico e social (Minayo et al., 2000).

A multidimensionalidade da qualidade de vida diz respeito ao fato de que o conceito é composto por diferentes dimensões, com abordagens gerais e holísticas, como fica evidenciado na definição da Organização Mundial de Saúde (OMS, 1995, p. 1405): "Qualidade de vida é a percepção do indivíduo sobre a sua posição na vida, no contexto da cultura e dos sistemas de valores nos quais ele vive, e em relação a seus objetivos, expectativas, padrões e preocupações". A definição é centrada na percepção subjetiva das diversas dimensões, que incluem saúde física, estado psicológico, nível de independência, interações sociais com o meio ambiente e crenças religiosas, entre outros tantos aspectos que podem ser derivados da definição.

$\mathrm{Na}$ atualidade, com o aprimoramento das pesquisas, os termos "qualidade de vida" e "qualidade de vida e saúde" ganham fundamentação teórica e metodológica (Seidl \& Zannon, 2012 ; Vilarta et al., 2010), incluindo um arcabouço de questões voltadas para a promoção da saúde com demandas específicas, tais como envelhecimento humano (Freitas \& Scheicher, 2010; Saccomann, Cintra, \& Gallani, 2011), qualidade de vida no trabalho (Klein, Lemos, Pereira, \& Beltrame, 2017; Pizzio \& Klein, 2015; Sanchez, Sanchez, Barbosa, Guimarães, \& Porto, 2019) e doenças crônicas e degenerativas (Almeida, Silveira, Santo, Pereira, \& Salomé, 2013; Kepla et al., 2013; Saço, Paula, Migliorini, Pereira, \& Ferreira, 2011; Souza et al., 2012).

\subsection{Descrever a lógica do conceito}

Há uma taxonomia das definições sobre qualidade de vida relacionada às épocas do desenvolvimento do conceito. A do Tipo I se refere a Definição global (até os anos 1980); a do Tipo II se refere a Definição com base em componentes (nos anos 1980); a definição do Tipo III trata das Definições focalizadas (1995); e a do Tipo IV trata das Definições operacionais (após 1995) (Farquhar, 1995).

As primeiras eram gerais, não identificavam as dimensões específicas do conceito, e thes faltava operacionalidade, por centralizarem-se em avaliações dicotômicas do tipo satisfação 
versus não satisfação com a vida. Elas variam principalmente pelo enfoque bioético ou de saúde pública.

As definições baseadas em componentes surgem nos anos 1980, com a operacionalização do conceito, e são classificadas por Farquhar (1995) como Tipo II - Definição com base em componentes: habilidades funcionais ou de saúde, apoiadas em aspectos empíricos; Tipo III - Definição focalizada: uma subdivisão das definições por componentes; e Tipo IV Definição das combinações, que ilustra o momento atual, incorporando aspectos dos Tipos II e III e favorecendo aspectos globais em diversas dimensões. O Tipo IV dá ênfase aos aspectos operacionais e possibilita a criação de instrumentos que avaliam tanto o aspecto global como o fatorial.

Os instrumentos que medem a qualidade de vida relacionada à saúde são classificados em dois grupos: genéricos e específicos (Rôla, Silva, \& Nicola, 2018).

Os genéricos avaliam os conceitos de saúde e representam valores humanos básicos, sendo relevantes para o estado de saúde e bem-estar de qualquer pessoa. Os itens não estão diretamente relacionados a nenhuma doença específica, podendo ser aplicados em indivíduos sadios e em todos os tipos e graus de severidade de doença, em tratamentos médicos diferentes e entre sub-grupos culturais e demográficos (Gordia et al., 2011). Eles permitem a comparação dos níveis de comprometimento da qualidade de vida entre diferentes doenças, bem como a comparação dos níveis de rebaixamento de grupo de doentes em relação à população geral (Rebollo \& Ortega, 2002). Mensuram aspectos funcionais, físicos, dor, estado geral de saúde, vitalidade, aspectos sociais, emocionais e saúde mental (Aguiar et al., 2008).

Os específicos são focados nos problemas associados a uma determinada doença, ou a áreas de função mais comumente afetadas em um grupo de pacientes (Gordia et al., 2011; Kutlay et al., 2003). Para Laupacis, Muirhead, Kneown e Wong (1992), a sua principal limitação é não ser adequado para comparar qualidade de vida entre grupos com especificidades terapêuticas distintas; por exemplo, entre hemodialisados e transplantados. Entretanto, sua sensibilidade descritiva (detectação de mudanças) é maior do que dos genéricos, já que os itens envolvem questões específicas de uma determinada doença questões estas que podem não ser avaliadas por um instrumento genérico. Por não haver instrumento ideal, alguns preconizam o uso combinado de genéricos e específicos, culminando nos chamados "modulares" (Dew \& Simmons, 1990). 


\subsection{Diferenciar as relações entre as perspectivas}

Segundo Pereira, Teixeira e Santos (2012), o termo "qualidade de vida" se apresenta na literatuta de forma diversificada. Devido à falta de consenso teórico e metodológico, pesquisas preferem utilizar conceitos como bem-estar, estilo de vida e saúde como sinônimos de qualidade de vida.

Em quaisquer das definições de qualidade de vida há sempre como incluir o fator saúde. Saúde não é um termo de fácil definição, e tem se transformado ao longo da história. A definição adotada pela Organização Mundial da Saúde (OMS) desde 1948 diz que "saúde é o estado de completo bem-estar físico, mental e social, e não apenas a ausência de doença", e tem significado desde então um marco conceitual. A evolução do conceito de saúde é apontada nas conferências internacionais sobre Promoção da Saúde, de Ottawa (OMS, 1986) até Nairóbi (OMS, 2009).

Autores como Ferraz e Segre (1997) acusam a definição da OMS de ser "irreal, ultrapassada e unilateral" (p. 539), por considerarem que a condição perfeita é uma utopia, algo não desejável para um conceito que serve de parâmetro para decisões médicas individuais e de saúde pública. Cleary, Wilson e Fowler (1995) argumentam que o termo "refere-se aos vários aspectos da vida de uma pessoa que são afetados por mudanças no seu estado de saúde, e que são significativos para a sua qualidade de vida" (p. 91).

Smith, Avis e Assman (1999), por meio de meta-análise de 12 estudos com amostras de portadores de enfermidades crônicas como câncer, hipertensão e SIDA, entre outras, concluíram que a percepção da qualidade de vida é determinada pelas dimensões de saúde mental e bem-estar psicológico, enquanto a percepção do estado de saúde é determinada pela dimensão do funcionamento físico, que inclui variáveis como energia, fadiga e dor.

De acordo com Buss (2000), embora saúde e qualidade de vida sejam utilizados como sinônimos, são conceitos que apresentam especificidades, mas também uma relação entre si. Faz-se necessário, ainda, distinguir "qualidade de vida relacionada à saúde" de "estado de saúde", embora ambos sejam utilizados comumente na literatura como sinônimos (Guyatt, Feeny, \& Patrick, 1993).

Gill e Feinstein (1994) afirmam que "qualidade de vida relacionada à saúde" e "estado subjetivo e saúde" são termos relacionados à avaliação subjetiva do paciente e ao impacto no seu estado de saúde. Guiteras e Bayés (1993) definem "qualidade de vida relacionada à 
saúde" como "a valoração subjetiva que o paciente faz de diferentes aspectos de sua vida, em relação ao seu estado de saúde" (p. 179). Ebrahim (1995) define "qualidade de vida relacionada à saúde" como "o valor atribuído à duração da vida, modificado pelos prejuízos, estados funcionais e oportunidades sociais que são influenciados por doença, dano, tratamento ou políticas de saúde" (p. 1384).

Apesar da complexidade do assunto, Valderrábano, Jofre e López Gomes (2001) vêem a questão de forma mais simples: dizem que, quando se referem a qualidade de vida de enfermos de doenças crônicas, estão fazendo referência a qualidade de vida relacionada à saúde. É artificial o limite imposto pelo pesquisador: o termo "saúde" acoplado à qualidade de vida continua a ser multidimensional, mesmo quando se estuda a qualidade de vida relacionada à saúde (Muldoon, Barger, Flory, \& Manuck, 1998).

Todavia, continua a existir o problema sobre quem é o melhor sujeito para responder aos instrumentos. Slevin, Plant, Lynch, Drinkwater e Gregory (1988), no século passado, no início do incremento da medida de qualidade de vida, questionavam, no título de seu artigo: Who should measure quality of life, the doctor or the patient? ("Quem deve medir a qualidade de vida, médico ou paciente?"). Essa é uma questão repetida em nosso século, no auge da utilização das medidas de qualidade de vida na área médica, como colocado no artigo de Addington e Kalra (2001), intitulado Who should measure quality of life ("Quem deve medir a qualidade de vida").

\subsection{Identificar e examinar as proposições (hipóteses)}

É consenso entre pesquisadores (Bombardier \& Tugwell, 1987; Valderrábano et al., 2001; Vilarta et al., 2010) que questionários ou formulários são os instrumentos habituais para se medir a qualidade de vida. A complexidade do problema está na formatação e seleção para inclusão dos itens relevantes. Na confecção dos itens de um instrumento, pode-se consultar no primeiro momento alguns pesquisadores do tema; no segundo momento, deve-se eliminar a co-linearidade e a redundância e obter uma combinação de um número mínimo de variáveis que possa ter representatividade estatística da dimensão da qualidade de vida a ser estudada.

Muldoon et al. (1998) fazem observações a esse tipo de instrumento, criticando a transformação de vários aspectos e componentes da qualidade de vida em valores quantitativos. Segundo os autores, esses componentes podem ser divididos em 3 principais 
domínios: físico, psicológico e social. No domínio físico estão incluídos aspectos como capacidade funcional e capacidade laborativa; no psicológico, incluem-se a satisfação, o bem-estar, a auto-estima, a ansiedade e a depressão; no domínio social, a interação com a família, amigos e ambiente de trabalho. A maioria desses componentes não pode ser observada diretamente. A forma usual de avaliação baseia-se na teoria da medida-do-item, uma medida indireta de um valor através das respostas do paciente a uma série de questões, denominadas "itens".

Bombardier e Tugwell (1987) delimitaram as questões mais pertinentes no desenho de um instrumento para medição de qualidade de vida relacionada à saúde, destacando como pontos principais: clareza dos objetivos, determinação da função do instrumento, metodologia para seleção de perguntas e escolha do conteúdo, forma de resposta, plausibilidade biológica e exequibilidade dos instrumentos. Valderrábano et al. (2001) dividem as propriedades necessárias para validar um instrumento de medida de qualidade de vida em: a) propriedades psicométricas (sensibilidade, validade interna, reprodutibilidade) e b) propriedades clínicas (facilidade de entendimento, tempo curto de preenchimento, validação cultural).

Muldoon et al. (1998) sublinham que a função do instrumento é, basicamente, discriminar, descrever e predizer o nível de qualidade de vida dos indivíduos escolhidos para o estudo. A propriedade discriminativa permite estabelecer diferenças entre os doentes de uma mesma enfermidade, sem sofrer influências de variáveis não relacionadas à saúde - como, por exemplo, a altura do indivíduo. O ideal seria a validade discriminativa total, mas, na sua impossibilidade, o reconhecimento de fatores de confusão faz com que sejam utilizadas as devidas ponderações na interpretação dos resultados.

A função descritiva se refere à possibilidade de o instrumento ser utilizado durante a evolução de uma enfermidade, detectando as mudanças que possam ocorrer; a função preditiva aborda o nível de comprometimento futuro (desfechos clínicos não-satisfatórios) da qualidade de vida; e a terceira função, a preditiva, permite decidir pela intensidade de tratamentos e intervenções no tempo presente (Muldoon et al., 1998).

Autores como Bombardier e Tugwell (1987) apontam outras formas da validação dos instrumentos, quais sejam: validade convergente e validade relacionada à exatidão dos resultados (acurácia). 
A validade convergente é testada quando se pode comparar os resultados de um aspecto com outro instrumento que faria o papel de padrão-ouro. Por exemplo, a pontuação referente ao bem-estar psicológico deve se relacionar à escala de outro instrumento validado para medida de sintomas depressivos. Outra possibilidade seria a comparação com questionários preenchidos por profissionais responsáveis pelo cuidado do paciente ou dos familiares - ainda que, para alguns autores, os aspectos subjetivos sejam os únicos itens que devam constar em uma pesquisa sobre qualidade de vida (Gill \& Feinstein, 1994). Essa postura deriva do entendimento de que o paciente tem acesso privilegiado e importância central à medida da qualidade de vida proporcionada pela doença e/ou terapia. Para estes aspectos, fortemente subjetivos, fica difícil a validação convergente que exige comparação (Bombardier \& Tugwell, 1987).

A validade relacionada à acurácia (exatidão) dos resultados depende das habilidades cognitivas e de comunicação do pesquisado, o que constitui uma das fragilidades mais comumente apontadas pelos críticos da validade deste tipo de pesquisa. A acurácia do instrumento fica então prejudicada na presença de transtornos do poder cognitivo, que interferem na informação correta e no julgamento sobre questões que envolvem, principalmente, o domínio da memória, do trabalho, do sexo e do envolvimento familiar. Os críticos deste tipo de instrumento chamam a atenção para o fato de que não é comum a realização de testes sobre o nível cognitivo dos pesquisados ou mesmo de testes observacionais, quando da validação dos mesmos (Bombardier \& Tugwell, 1987).

Os instrumentos devem ter a qualidade científica da reprodutibilidade, pois os dados obtidos ao longo da medição devem ser consistentemente repetidos pelo mesmo observador ou por outros. Podem ser utilizados os métodos de teste-reteste durante um intervalo curto 0 bastante para que não haja mudança do estado de saúde do sujeito pesquisado (Bombardier \& Tugwell, 1987).

O instrumento deve ter também plausibilidade biológica. Quanto mais a medição do questionário se correlacionar com os estados clínicos, mais credibilidade na área médica ele terá. Por exemplo, um questionário sobre a capacidade funcional pode ser comparado com testes objetivos como caminhar, sair da posição sentada para a de ficar em pé etc. Porém, ainda se conhece pouco sobre as relações entre esses componentes; permanece o aprimoramento metodológico do constructo "qualidade de vida e saúde". Algumas propriedades são essenciais para que o questionário se torne prático: aplicá-lo em curto 
tempo, para a exequibilidade; validá-lo culturalmente; e não se limitar à tradução dos seus itens, que devem ser entendidos por toda a população pesquisada (Bombardier \& Tugwell, 1987).

\section{CONCLUSÕES}

Dentre as muitas expressões conceituais analisadas neste estudo, toma-se evidente a importância da medida de qualidade de vida e saúde, que são adotadas como sinônimos. A especificidade dessa medida faz com que a validade possa ser bem testada em alguns aspectos e apresente dificuldade em outros, como discutido neste estudo. A medida da qualidade de vida depende de mais consenso entre os especialistas e da segurança sobre a validade científica dos instrumentos de coleta de dados, seja ele genérico ou específico. Permanece sendo necessário o debate sobre o constructo "qualidade de vida e saúde".

\section{REFERÊNCIAS}

Addington, J., \& Kalra, L. (2001). Who should measure quality of life? British Medical Journal, 322(7299), 14171420.

Aguiar, C. C. et al. (2008). Instrumentos de Avaliação de Qualidade de Vida Relacionada à Saúde no Diabetes Melito. Arquivos Brasileiros de Endocrinologia \& Metabologia, 52(6), 931-939.

Almeida, S. A., Silveira, M. M., Santo, P. F., Pereira, R. C., \& Salomé, G. M. (2013). Assessment of the quality of life of patients with diabetes mellitus and foot ulcers. Revista Brasileira Cirurgia Plástica, 28(1), 142-146.

Bombardier, C., \& Tugwell, P. (1987). Methodological considerations in functional assessment. Journal Rheumatology Supplement, 15(2), 6-10.

Brasel, K. J. (2007). Quality of life assessment in palliative care. Surgery Palliative Care, 24(3), 231-235.

Buss, P. M. (2000). Promoção da saúde e qualidade de vida. Ciência \& Saúde Coletiva, 5(1), 163-177.

Caron, C. D., \& Bowers, B. J. (2000). Methods and application of dimensional analysis: a contribution to concept and knowledge development in nursing. In B. L. Rodgers \& K. A. Knafl. (Eds.). Concept development in nursing: foundations, techniques, and applications (pp. 285-319). Philadelphia: Saunders Company.

Cleary, P. D., Wilson, P. D., \& Fowler, F. J. (1995). Health-related quality of life in HIV-infected persons: a conceptual model. In F. E. Dimsdale \& A. Baum. (Eds.). Quality of life in behavioral medicine research (pp. 191-204). New Jersey: Lawrence Erlbaum Associates.

Costa, B. B. N., \& Araújo, T. C. C. F. (2001). Avaliação da qualidade de vida em pacientes com câncer de cabeça e pescoço. Temas da Psicologia, 9(2), 128-135.

Dew, M. A., \& Simmons, R. G. (1990). The advantage of multiple measures of quality of life of quality of life. Scandinavian Journal of Urology and Nephrology, 24(131), 23-30.

Ebrahim, S. (1995). Clinical and public health perspectives and applications of health-related quality of life meausurement. Social Science \& Medicine, 41(10), 1383-1394.

Farquhar, M. (1995). Definitions of quality of life: a taxonomy. Advanced Nursing, 22(3), 502-508.

Fayers, P. M. O. (2000). Quality of life assessment, analysis and interpretation. Chichester: John Wiley. 
Ferraz, F. C. O., \& Segre, M. (1997). Conceito de saúde. Saúde Pública, 31(5), 538-542.

Freitas, M. A. V., \& Scheicher, M. E. (2010). Qualidade de vida de idosos institucionalizados. Revista Brasileira Geriatria Gerontologia, 13(3), 395-401.

Gaiva, M. A. M. (1998). Qualidade de vida e saúde. Revista de Enfermgem UERJ, 6(2), 377-382.

Garratt, A., Schmidt, L., Mackintosh, A., \& Fitzpatrick, R. (2002). Quality of life measurement: bibliographic study of patient assessed health outcome measures. British Medical Journal, 324(7351), 1417-1429.

Gill, T. M., \& Feinstein, A. R. (1994). A critical appraisal of the quality of quality-of-life measurements. Journal of the American Medical Association, 272(8), 619-626.

Gordia, A. P. et al. (2011). Qualidade de vida: contexto histórico, definição, avaliação e fatores associados. Revista Brasileira de Qualidade de Vida, 3(1), 40-52.

Guiteras, A. F., \& Bayés, R. (1993). Desarrollo de un instrumento para la medida de la calidad de vida en enfermidades crónicas. In M. Forns \& M. T. Anguera. (Eds.). Aportaciones recientes a la evaluación psicológica (pp. 175-195). Barcelona: Universitas.

Guyatt, G. H., Feeny, D. H., \& Patrick, D. L. (1993). Measuring health-related quality of life. Annals Internal Medicine, 118(8), 622-629.

Kepla, S. et al. (2013). The relationship between traits optimism and anxiety and health-related quality of life in patients hospitalized for chronic diseases: data from the SATISQOL study. Health and Quality of Life Outcomes, 11(134), 1-12.

Klein, L. I., Lemos, R. B., Pereira, B. A. D, \& Beltrame, G. (2017). Qualidade de vida no serviço público: uma avaliação em uma instituição de ensino superior. Revista de Administração, 23(esp.), 317-344.

Kutlay, S. et al. (2003). General or disease specific questionnaire: a comparative study in hemodialysis patients. Renal Failure, 25(1), 99-103.

Laupacis, A., Muirhead, N., Kneown, P., \& Wong, C. (1992). A disease-specific questionnaire for assessing quality of life in patients on hemodialysis. Nephron, 60(3), 302-306.

Minayo, M. C. S., Hartz, Z. M. A., \& Buss, P. M. (2000). Qualidade de vida e saúde: um debate necessário. Ciência \& Saúde Coletiva, 5(1), 7-18.

Muldoon, M. F., Barger, S., Flory, J. D., \& Manuck, S. B. (1998). What are quality of life measurements measuring? British Medical Journal, 316(7130), 542-545.

OMS [Organização Mundial da Saúde]. (2009). The Nairobi call to action for closing the implementation gap in health promotion. Geneva: OMS Press.

OMS [Organização Mundial da Saúde]. (1995). Quality of life assessment: position paper from the World Health Organization. Social Science Medicine, 41(10), 1403-1409.

OMS [Organização Mundial da Saúde]. (1986). Carta de Ottawa para a promoção da saúde: primeira conferência internacional sobre promoção da saúde. Genebra: OMS Press.

Pereira, E. F., Teixeira, C. S., \& Santos, A. (2012). Qualidade de vida: abordagens, conceitos e avaliação. Revista Brasileira de Educação Física e Esporte, 26(2), 241-50.

Pizzio, A., \& Klein, K. (2015). Qualidade de vida no trabalho e adoecimento no cotidiano de docentes do Ensino Superior. Educação \& Sociedade, 36(131), 493-513.

Rebollo, P., \& Ortega, F. (2002). New trends on health related quality of life assessment in end-stage renal disease patients. International Urology and Nephrology, 33(1), 195-202. 
Rôla, C. V. S., Silva, S. P. C., \& Nicola, P. A. (2018). Instrumentos de avaliação da Qualidade de Vida de pessoas jovens e idosas: um estudo de Revisão Sistemática. Revista Multidisciplinar de Psicologia, 12(42), 111-120.

Saccomann, I. C. R., Cintra, F. A., \& Gallani, C. B. J. (2011). Qualidade de vida relacionada à Saúde em Idosos com Insuficiência cardíaca: avaliação com instrumento específico. Acta Paulista de Enfermagem, 24(2), 179-184.

Saço, L. F., Paula, O. R., Migliorini, G. E., Pereira, N. P., \& Ferreira, E. L. (2011). Características e avaliação da qualidade de vida em um grupo de pacientes submetidos a tratamento quimioterápico. Hospital Universitário, 37(1), 95-102.

Sanchez, H. M., Sanchez, E. G. M., Barbosa, M. A., Guimarães, E. C., \& Porto, C. C. (2019). Impacto da saúde na qualidade de vida e trabalho de docentes universitários de diferentes áreas de conhecimento. Ciência \& Saúde Coletiva, 24(11), 4111-4123.

Seidl, E. M. F., \& Zannon, C. M. L. C. (2004). Qualidade de vida e saúde: aspectos conceituais e metodológicos. Cadernos de Saúde Pública, 20(2), 580-588.

Slevin, M. L., Plant, H., Lynch, D., Drinkwater, J., \& Gregory, W. M. (1998). Who should measure quality of life, the doctor or the patient? British Journal of Cancer, 57, 109-112.

Smith, K. W., Avis, N. E., \& Assman, S. F. (1999). Distinguishing between quality of life and health status in quality of life research. Quality Life Research, 8(5), 447-459.

Souza, E. C. S. et al. (2012). Avaliação da qualidade de vida de portadores de diabetes utilizando a medida específica B-PAID. Revista Mineira de Enfermagem, 16(4), 509-514.

Valderrábano, F., Jofre, R., \& López Gomez, J. M. (2001). Quality of life in end-stage renal disease patients. American Journal of Kidney Diseases, 38(3), 443-464.

Viana, A. G., \& Sampaio, L. (2019). Qualidade de vida dos universitários em período de conclusão de curso. Revista Multidisciplinar de Psicologia, 13(47), 1085-1096.

Vilarta, R., Guitierrez, G. L., \& Monteiro, M. I. (2010). Qualidade de vida: evolução dos conceitos e práticas no século XXI. Campinas: Ipes. 